\title{
RECENT STATUS OF VERY HIGH ENERGY GAMMA RAY ASTRONOMY AND PROSPECTS
}

\author{
T. KIFUNE \\ Institute for Cosmic Ray Research, University of Tokyo, \\ Kashiwa-no-ha 5-1-5, Kashiwa, Chiba 277-8582, Japan \\ E-mail: tkifune@icrr.u-tokyo.ac.jp
}

\begin{abstract}
Gamma-rays, located at the highest energy band of electromagnetic radiation, is directly coupled to the behaviour of energetic particles created and accelerated in the Universe, which are known as cosmic rays. The information on these energetic non-thermal particles, protons in particular, is hard to obtain through other bands. The results of very high energy $\gamma$-rays are summarized by paying a special attention to the origin of cosmic rays.
\end{abstract}

\section{Introduction}

Observation of VHE (very high energy) $\gamma$-rays in $100 \mathrm{GeV}-10 \mathrm{TeV}$ region has been developed ${ }^{1}$ during the last decade of years, successful to detect $\mathrm{TeV}$ $\gamma$-ray radiation as summarized in Table 1 . Gamma-rays are a direct probe of cosmic high energy particles, the products of the non-thermal processes of the Universe. The "origin of cosmic rays" is still not fully clarified, addressing questions in the basic aspects of high energy processes in the Universe; (i) "Acceleration sites or point sources of cosmic rays?", (ii) "Propagation of cosmic rays or the high energy structure of the Galaxy?", and (iii) "Unknown aspect of particle physics or new enigmata?". High energy $\gamma$-rays can be a good probe of cosmic rays at far distances, and one of the scientific goals of $\gamma$-ray astronomy is to provide key to answer the questions above.

Emission from the Galactic disc is the brightest of $\mathrm{GeV} \gamma$-rays ${ }^{2,3}$, and provides evidence for protons certainly accelerated, filling the Galactic disc, and colliding with interstellar matter to produce $\gamma$-rays. VHE $\gamma$-rays from the known point sources are explained, however, by electron progenitor, rather than by protons which are the main constituent of cosmic rays, and the acceleration site of protons remains to be looked for. Thus, it still remains to study into more details of cosmic rays escaping from the acceleration sites, propagating in the Galactic disc and then flowing out of the Galaxy.

Blazars, $\gamma$-ray emitting AGN (active galactic nuclei), have extended beyond our Galaxy the horizon of viewing the energetic non-thermal processes of the Universe. Detection of $\gamma$-rays or cosmic rays of other galaxies gives us a new insight for understanding our own Galaxy. Cosmic rays are observed up to $\sim 10^{20} \mathrm{eV}$, and such high energy particles are believed to be of extragalactic 
origin. Relativistic jets and fire balls of AGN and GRB (gamma ray burst) are speculated to be the site of most energetic cosmic rays up to $10^{20} \mathrm{eV}$.

Table 1: Characteristics of $\mathrm{TeV} \gamma$-ray sources in comparison with $\mathrm{GeV}$ sources

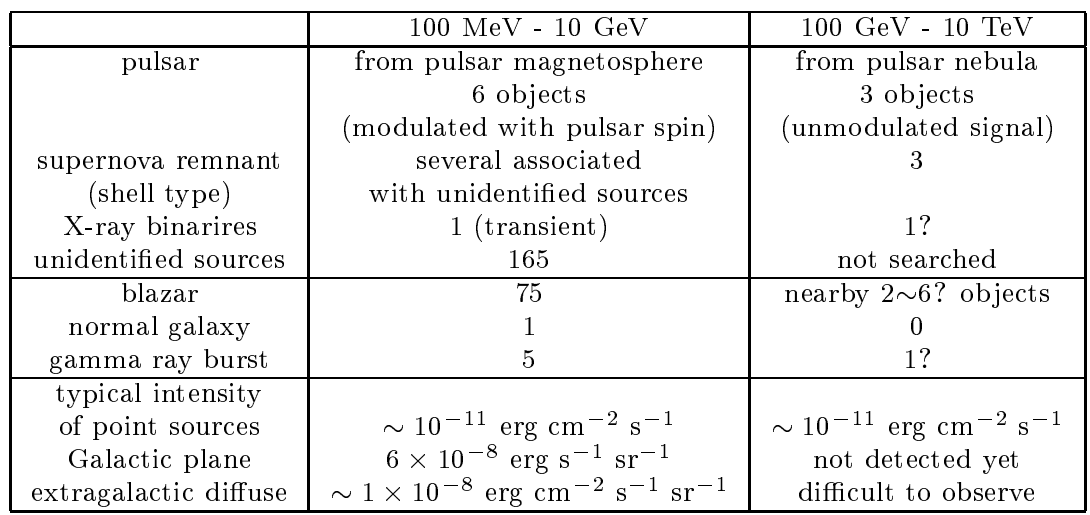

VHE $\gamma$-ray astronomy was founded on the observational base provided by the technique of the IACT (imaging air Čerenkov telescope). The telescope observes Cerenkov lights from extensive air shower initiated by $\gamma$-rays. The lights spread with an aperture of $\sim 1^{\circ}$ around the incoming direction of $\gamma$-rays illuminating the area of $\sim 0.1 \mathrm{~km}^{2}$ on the ground. This huge detection area provides good statistics of $\gamma$-ray photons in VHE region and the study of the image structure of the lights enables to achieve angular resolution of $\sim 0.1^{\circ}$ as well as reduction of overwhelming background of cosmic ray charged particles. The detection area of $\sim 0.1 \mathrm{~km}^{2}$ gives, with several tens of hours of observation time, a sensitivity of $\sim 10^{-12} \mathrm{erg} \mathrm{cm}^{-2} \mathrm{~s}^{-1}$ for detecting VHE $\gamma$-ray flux. A further step of improvement is expected to be taken by a system of multiple IACTs of the Projects, VERITAS, CANGAROO, and HESS Collaborations, aiming at; the threshold energy of detecting $\gamma$-rays to be reduced as low as $\sim 100 \mathrm{GeV}$, allowing discrimination of the cosmic ray background and better angular and energy resolution, by simultaneous, stereoscopic operation of the multiple telescopes. However, the field of view of IACT is limited to $\sim 0.01$ sr, and independent operation of many telescopes will be used to search in a wider area of sky. 


\section{TeV Point Sources}

SNR (super nova remnant) is the most likely candidate of the sites of proton acceleration, and are, among a variety of $\gamma$-ray sources, of the prime interest in the context of understanding the origin of cosmic rays. The $\gamma$-ray emission from proton progenitor is expected in the both bands of $\mathrm{GeV}$ and $\mathrm{TeV}$ energy. However, no SNR is yet detected as a common source of both $\mathrm{GeV}$ and TeV bands. Cosmic rays beyond the knee energy of $\sim 10^{15} \mathrm{eV}$ is hardly explained by SNRs and other types of acceleration sites are argued for a long time.

\subsection{Supernova Remnant, Pulsar and Nebula}

Six pulsars are known to be $\mathrm{GeV} \gamma$-ray sources. All of these pulsars show pulsed or time-modulated signal with the pulsar spin period. The pulsed signal is explained by the copious pair plasma of energetic electrons and positrons in the pulsar magnetosphere which co-rotates with neutron star. The pulsed emission disappears with increased energies, and in the $\mathrm{TeV}$ energies, unpulsed emission emerges dominant. The pulsar wind blows out of the magnetosphere, collide with the circumferential matter to form pulsar nebula, and is considered to radiate $\mathrm{TeV} \gamma$-rays. Thus, the emission mechamism of the $\mathrm{GeV}$ and $\mathrm{TeV}$ sources are disimilar to each other, except for the Crab nebula, the only known pulsar that has unpulsed signal also at GeV energy.

The Crab pulsar was given birth as a product of supernova explosion a thousand years ago. The pulsar dominates over the activity of the explosion ejecta that forms at present a shell-like structure of SNR. The pulsar wind is interpreted as a relativistic flow of the pair plasma of electrons and positrons, and thus, it is not surprising that the emission is dominated by the electron progenitor and not by protons, as evidenced by the Spectral Energy Distribution (SED) over the "entire" bands consisting of two components of synchrotron and inverse Compton radiations. Radiation from protons is hidden, if exists, below the intense contribution from the electron progenitor. However, electrons will cease to be accelerated, due to the rapid radiation loss, at lower energies than protons. Evidence for the proton progenitor would be possibly found in the energy spectrum beyond $\mathrm{TeV}$ energies if studied into more details.

Shell-type SNR that lacks the pulsar activity at the center of the remnant is a good candidate for the objects from which there appear clearly the $\gamma$-rays emitted from protons that are shock-accelerated in the supernova shell. Several unidentified sources at $\mathrm{GeV}$ energy are associated with shell-type SNRs within the error circle of anglular resolution ${ }^{4}$. The attempt for $\mathrm{TeV} \gamma$-rays from those objects has been so far not successful and the upper limit of $\mathrm{TeV}$ gamma ray flux is below the flux extrapolated from the $\mathrm{GeV}$ intensities. Evidence of $\mathrm{TeV} \gamma$ - 
ray emission was found, however, from three SNRs, SN $1006^{5}$, RXJ 1713-395 ${ }^{6}$, and Cas $\mathrm{A}^{7}$ that are not $\mathrm{GeV}$ sources. The apparent SED over the wide band from $100 \mathrm{MeV}$ to $1 \mathrm{TeV}$ is explained by two contributions of synchrotron and inverse Compton radiations of electrons, thus supporting the model of electron progenitor. Based on the fact that the environmental matter density is as low as 0.1 proton $\mathrm{cm}^{-3}$, the result of SN 1006 suggests that: The maximum acceleration energy $E_{\max }$ anticorrelates with Luminosity and thus the less matter density of SNR environments leads to higher acceleration energy ${ }^{8}$. The period of SNR accelerating cosmic rays to higher energies is limited to earlier time of $\sim 1000$ years since ambient neutral atoms tend to damp Alfven wave in collision with ions and to reduce the acceleration efficiency ${ }^{9}$. The path to clarifying the origin of cosmic rays was not, thus, so simple and straightforward as we had expected.

\subsection{Active Galactic Nuclei (TeV Blazars)}

The SED from blazars is explained also by synchrotron and inverse Compton mechanism of electrons. As evidenced by the apparent superluminal jet of a blazar 3C273, the first discovered one. The intense $\gamma$-rays from blazars are allowed by the relativistic jet which is ejected toward us from the central engine presumably of a massive black hole in AGN. The geometry causes the enhancement of energy and intensity of $\gamma$-rays, explaining the unexpectedly large number of blazars discovered by EGRET against the $\gamma$-ray absorption, which is expected to occur in the emission region unless the jet maechanism works.

Interestingly, the TeV blazars are weak sources of the $\mathrm{GeV}$ band, and these appear disimilar to each other. The maximum energy of $\gamma$-ray photons from the various blazars appears to anti-correlate with the bolometric brightness. Such a feature of the observed SED is explained by the maximum acceleration energy $E_{e}^{\max }$ of electrons decreasing with the brightness of blazar, as it relates to the energy density $W$ of radiation and magnetic field as $E_{e}^{\max } \propto W^{-0.5}$, suggesting that the radiation loss controls the maximum energy of acceleration ${ }^{10}$. When blazars is bright or the jet is exposed to very intense external radiation field, Compton scattering of the external photon takes place to efficiently produce $\gamma$-rays, and limits $E_{e}^{\max }$ to give $\mathrm{GeV}$ Blazars. If it is less bright, the target photons of Compton scattering need to be served by own self, i.e. by synchrotron radiation from the jet, and we have TeV Blazars.

Although the observed data are fairly well explained by electrons, the case of a jet that consists of protons, Hadron Jet model ${ }^{11}$, is attractive to explain the origin of extragalactic cosmic rays. Recently, attempts ${ }^{12}$ are made 
to attribute the higher energy component of SED to proton's synchrotron radiation, not to the electron's inverse Compton radiation, and the lower energy one to the cascade process initiated by energetic protons. The energy $\epsilon$ of synchrotron photons from protons of energy $E_{p}$ is given by $\epsilon \approx(B / 1000 G)$. $\left(E_{p} / 10^{19} \mathrm{eV}\right)^{2} \mathrm{TeV}$. Thus, the hadron jet model leads to a possibility that blazars are a likely source of cosmic rays of ultra high energies.

There are several hurdles to overcome before we can test the models of the lepton or hadron jet by comparing observed SED with calculations. Since blazars are highly time-variable, the "entire" SED over multi bands need to be determined from simultaneous observations. Another uncertainty stems from the opacity, which is due to the pair production when $\gamma$-rays collide with infrared photons in the extragalactic space, and the observed SED requires correction for the absorption effect.

A TeV blazar, Mkr 501, have shown, during the outburst in 1997, an energy spectrum extending up to $\sim 20 \mathrm{TeV}$. The intrinsic SED after the correction ${ }^{13,14}$ yields a spectrum of a power index $\approx 2$ or even much flatter when intenser infrared intensity is adopted. Such a hard spectrum might prefer proton progenitor, and if so the hidden luminosity can be enormous in higer energy region. The extragalactic intensity of infrared photons, which is importnat to infer the epoch of galaxy/star formation is also crucial when we argue about the hadron jet and even speculation of some exotic processes.

\subsection{Gamma Ray Burst, Unidentified $\gamma$-ray Sources and New Classes of TeV sources}

Five GRBs (gamma ray bursts) have been detected by EGRET showing an energy spectrum extending at least up to $\mathrm{GeV}$ energies. The limited field of view $\sim 10^{-2} \mathrm{sr}$ of IACT makes it difficult in TeV region to observe the objects of such violent time variability. The Milagrrito experiment of ground-based water Čerenkov detector and of having a field of view $\sim 1$ sr, has reported ${ }^{15}$ a burst of events at high rate, at the time of GRB 970417a with a chance probability of coincident occurrence to be $1.5 \times 10^{-3}$, which might imply a detection of the GRB with luminosity $\sim 10^{-6} \mathrm{erg} \mathrm{cm}^{-2}$ at $\sim 1 \mathrm{TeV}$ energies.

The conventional candidate of origin of cosmic rays, SNR, is a stable source of $\gamma$-rays. If one per cent of explosion energy of supernova is used for acceleraton and then a further fraction of $\sim 10^{-5} \approx$ (confinement time in SNR region) / (mean collision time with interstellar matter) used for $\gamma$-ray emission during $10^{3}$ years, $\gamma$-ray luminosity of SNR woudl be $\sim 10^{34} \mathrm{erg} \mathrm{s}^{-1}$. Bright VHE sources have a flux of $\sim 10^{-11} \mathrm{erg} \mathrm{cm}^{-2} \mathrm{~s}^{-1}$, which corresponds to $\approx 10^{34}$ $\mathrm{erg} \mathrm{s}^{-1}$ at $3 \mathrm{kpc}$ distance. Thus, new, unknown populations of $\gamma$-ray sources 
would, if exist, be then likely to exhibit violent time variability of short time duration. In the $\mathrm{GeV}$ region, a majority of Galactic sources are still unidentified ${ }^{16}$. Several of them, such as emission from the Galactic center, have been found to be time variable ${ }^{17}$.

A possibility of the relativistic shcok of GRB accelerating particles has been speculated as the origin of cosmic rays at ultra high energies. Galactic objects such as X-ray binaries and Galactic jet objects are known also to show violent time variability, and some of them are found to extend their emission at least into $\mathrm{MeV}$ energies ${ }^{18}$. High energy particles in the binary system have a chance of collision with ambient matter supplied by the companion star, and $\mathrm{TeV} \gamma$-ray production from proton-proton collision can be expected.

If protons are transiently accelerated to appear as highly time variable $\gamma$-ray sources, their activities are hard to be seen in other wavelengths like the case of Geminga pulsar which had remained long as a mysterious unidentified $\gamma$-ray source.

\section{Diffuse emission: Origin and Propagation of Cosmic Rays}

Cosmic ray protons suffer from energy loss in collision with interstellar matter during propagation with the characteristic time being at least $\sim 10^{8}$ years (for matter density of $\sim 1$ proton per cubic centimeter). The confinement time of cosmic rays in the Galaxy is shorter by an order of magnitude, and the propagation of cosmic rays reveals the structure of the Galactic disc by $\gamma$-rays emitted through creation of $\pi^{0}$ meson.

\subsection{Galactic Disc Emission}

The "overall profile" of the origin of Galactic cosmic rays, integrated over various acceleration sites and propagation mechanism, would be found in the diffuse $\gamma$-rays from the Galactic plane. At energies higher than $\sim 1 \mathrm{GeV}$, the $\gamma$-ray intensity observed ${ }^{2}$ tends to be higher than the expected flux from the conventional model of cosmic ray protons, which is based on the spectrum of local cosmic rays in the vicinity of the solar system and is with an assumption that the cosmic ray intensity is in correlation with the matter density in the interstellar space.

The behaviour of cosmic rays, such as the confinement time in the Galaxy, is known with less uncertainty at lower energies. The discrepancy between observation and the model calculation increases, and theoretical models might be better tested in higher energy region: The contribution from SNR will increase from less than $10 \%$ at $\mathrm{GeV}$ energies to become dominant at energies higher than $100 \mathrm{GeV}^{19}$; possible change of the index of power of energy spectrum 
may appear if a new population of cosmic ray origin emerges, and the variation against Galactic latitude and longitude will suggest the global distribution of acceleration sites. At energies near and higher than $10^{15} \mathrm{eV}$, a larger scale of spatial size such as the shock by the Galactic wind may be the case for cosmic ray acceleration, which may predict the extended emission of $\gamma$-rays from the inner Galactic disc or the region near to the Galactic halo.

However, the Galactic disc emission have not been yet detected in the region of $\mathrm{TeV} \gamma$-rays ${ }^{20}$. The observation is not easy because the light image of IACT extends about $1^{\circ}$, the imaging technique of achieving $0.1^{\circ}$ is not easy to apply to extended sources and to reject the overwhelming cosmic ray background within the field of view of about $3^{\circ}$.

\subsection{Extragalactic (high latitudes) diffuse emission}

Detection of energetic $\gamma$-rays from the whole cosmic rays contained in normal galaxies, starburst galaxies and cluster of galaxies ${ }^{21}$ will be targets of the next generation of telescopes; a system of IACTs and GLAST.

In the extragalactic space, VHE $\gamma$-rays travel long enough to collide with infrared photons of cosmic background radiation and are converted into a pair of electron and positron. Thus, VHE $\gamma$-rays are constrained by their opacity in the extragalactic space from our neighborhood within distances $\sim 100 \mathrm{Mpc}$ at $\sim 1 \mathrm{TeV}$. Thus, the energy spectrum is likely to show in the TeV energy region a dramatic cutoff, which would tell us about the total yield of VHE $\gamma$-rays from nearby sources as well as the characteristic distance to the sources. The limited number of nearby sources possibly cause a considerable fluctuation of intensity. Although it is not easy to distinguish diffuse $\gamma$-rays from the cosmic ray background, the spectral feature and anisotropic intensity may allow us to infer the extragalactic diffuse $\mathrm{TeV} \gamma$-rays,

\section{Unknown phenomena: Particle interaction}

Detection of exotic processes have been and would still remain to be an interesting objective of $\gamma$-ray astronomy ${ }^{22}$. As one of such topics, a line emission of $\gamma$-rays at $100 \mathrm{GeV}$ to $\mathrm{TeV}$ energies may result from unkown dark matter which is presumably concentrated in the Galactic center. The observation is, however, with accuracy $\sim \Delta E / E \sim 20 \%$, the energy resolution of the current IACT technique, which needs to be largely improved. Another possiblility is to detect a burst of very short duration of $\gamma$-rays from transient sources like primordial black holes, as has been attempted by EGRET ${ }^{23}$. The detection of GRB or transient outbursts from unknown sources is one of the most urgent objectives for VHE $\gamma$-ray astronomy presently to aim at. Generally, unknown 
aspect of particle interaction would be expected in higher energy region, and detection of $\gamma$-rays beyond $\mathrm{TeV}$ energies requires new techniques different from IACT.

\section{Conclusion}

Pulsars, SNR and blazars are found to be the sources of VHE $\gamma$-rays. However, no $\gamma$-ray sources of proton progenitor have not been yet uncovered. The $\mathrm{TeV}$ sources appear disimilar to $\mathrm{GeV}$ sources, and this feature is well explained by radiation of electrons. Combined with the data in other bands, the strength of magnetic field is estimated from the ratio of inverse Compton to synchrotron intensiy. The consequenses are, as one of the multi-bands of entire SED, used to understand individual astronomical objects; acceleration of particles in the blazar jet or pulsar wind; environmental conditions of the enigmatic objects and so on.

In this paper, an emphasis was put on the aspect of VHE $\gamma$-ray astronomy that is closely coupled to cosmic ray physics. Shell-type SNRs remain yet to be confirmed as $\gamma$-ray sources of proton progenitor. The results from VHE $\gamma$-ray observation of SNRs imply that the efficiency and the maximum energy of acceleration would vary from object to object and a less number of SNRs contribute to cosmic rays of higher energies. The cosmic rays of energies between $10^{15}$ and $10^{19} \mathrm{eV}$ are usually believed to be of Galactic origin, and acceleration sites other than SNRs are also to be looked for possibly by using energetic $\gamma$-rays. AGN as well as GRB are interesting candidates for the origin of cosmic rays up to the maximum energies $\sim 10^{20} \mathrm{eV}$. The energy budget could be greatly altered, if protons are responsible for emission from these objects, for instance, the relativistic jet of blazars carrying an enormous amount of kinetic energy.

VHE $\gamma$-rays from other normal or active galaxies, as well as Galactic disc emission of $\mathrm{TeV} \gamma$-rays, would provide an unbiased view of the overall features of production and propagation of cosmic rays. The observable number of extragalactic VHE $\gamma$-ray sources are limited by infrared background radiation to be within $\sim 100 \mathrm{Mpc}$ proximity, and thus, it is interesting to extend our survey to $\mathrm{TeV}$ energies and even higher to know the existence of the spectral cutoff of the diffuse extragalactic gamma rays.

The small field of view of IACT has limited the TeV observation ever attempted to handful targets, and thus the known sources can be considerably biased for us to draw a fair view of VHE phenomena of the Universe. At least, objects of violent time variability as well as of extended emission have been slipped off from detection. Advanced observations are soon coming with the 
projects of a system of multiple IACTs, followed by the GLAST satellite, the all sky monitor for $100 \mathrm{MeV}-10 \mathrm{GeV} \gamma$-rays. However, VHE $\gamma$-rays requires their own all sky monitor ${ }^{24}$. Fig. 1 summarizes schematically the current status ans prospects.

Fig. 1. Energy spectra of cosmic ray, diffuse $\gamma$-rays from Galactic disc and high Galactic latitudes are plotted. The fluence in the vertical axis is in the unit of $\mathrm{eV} \mathrm{cm}^{-2} \mathrm{~s}^{-1}\left(10^{-3} \text { steradian }\right)^{-1}$ (i.e. plotted is extended emission per $10^{-3}$ steradian $\approx 1 / 10$ of IACT's field of view). The fluence of point-like source, GRB, as well as the IACT sensitivity, is also plotted in this figure, as if they have extension of $10^{-3}$ steradian, thus the unit in this case to be read as $\mathrm{eV} \mathrm{cm}^{-2} \mathrm{~s}^{-1}$. 


\section{References}

1. See for review, for instance, T.C. Weekes, Proc. of GeV-TeV Gamma Ray Astrophysics Workshop, ed. B.L. Dingus, M.H. Salamon and D.B. Kieda (AIP Conf. Proc. 515) , 3 (1999): references therein.

2. S.D. Hunter et al., Ap. J. 481, 205 (1997).

3. R.C. Hartman et al., Ap. J. Suppl 123, 79 (1999).

4. J.A. Esposito et al., Ap. J. 461, 820 (1996).

5. T. Tanimori et al., Ap. J. Lett. 497, L25 (1998).

6. H. Nuraishii et al., Astron. Astrophys. Lett. 354, L57 (2000).

7. G. Pühlhofer et al., Proc. 26th Cosmic Ray Conf. (Salt Lake City) 3, 492 (1999).

8. M.G. Baring et al., Ap. J. 513, 311 (1999).

9. L. O'C. Drury, J. Kirk, and P. Duffy, Astron. Astrophys 309, 1002 (1996).

10. G. Ghisellini et al., MNRAS 301, 451 (1998).

11. e.g. K. Manheim, W.M. Krülls, and P.L. Biermann, Astron. Astrophys. 251, 723 (1991).

12. A. Mücke and R.J. Protheroe, astro-ph/0004052 , (2000).

13. R.J. Protheroe and M. Meyer, astro-ph/0005349 , (2000).

14. J. Guy et al., astro-ph/0004355 , (2000).

15. J.E. McEnery et al., Proc. of GeV-TeV Gamma Ray Astrophysics Workshop, ed. B.L. Dingus, M.H. Salamon and D.B. Kieda (AIP Conf. Proc. 515) , 243 (1999).

16. N. Gehrels et al., Nature 404, 363 (2000).

17. P.M. Wallace et al., Ap. J. 540, 184 (2000).

18. V. Schönfelder et al., Astron. Astrophys. Suppl. 143, 145 (1999).

19. E.G. Berezhko and H.J. Völk, Ap. J. 540, 923 (2000).

20. S. Le Bohec et al., Ap. J. 539, 209 (2000).

21. H.J. Völk and A.M. Atoyan, Astroparticle Phys. 11, 73 (1999).

22. D.A. Kniffen and N. Gehrels, Proc. 4th Compton Symposium (AIP Conf. 410) 1, 524 (1997).

23. C.E. Fichtel and P. Sreekumar, Proc. 4th Compton Symposium (AIP Conf. 410) 1, 436 (1997).

24. T. Kifune, Proc. $\gamma$-ray Conference (Heidelberg); ICRR-Report-468-200012 (ICRR, Univ. of Tokyo) , (2000). 